\title{
Pyroelectric detectors based on high-performance PMN-PT single crystals
}

\author{
Shao Xiumei ${ }^{1}$, Ma Xueliang ${ }^{1,2}$, Fang Jiaxiong ${ }^{1}$ \\ ${ }^{1}$ Shanghai Institute of Technical Physics, Chinese Academy of Sciences, Shanghai 200083, China,
}

shaoxm@mail.sitp.ac.cn

${ }^{2}$ Graduate School of the Chinese Academy of Science, Beijing 100049, China

\begin{abstract}
:
Single crystal of $\mathrm{Pb}\left(\mathrm{Mg}_{1 / 3} \mathrm{Nb}_{2 / 3}\right) \mathrm{O}_{3}-\mathrm{PbTiO}_{3}$ (PMN-PT), as one of the best pyroelectric materials, was first discovered by Chinese scientist. In this paper, PMN-PT was selected as pyroelectric detector material. Firstly, an electrically calibrated pyroelectric detector with special structure was developed for calibration of radiation. The sensitive area of the detector is $10 \mathrm{~mm} \times 10 \mathrm{~mm}$ and the entrance aperture is $\Phi 8 \mathrm{~mm}$. The research about the coating film on the surface of the sensitive area was carried out. Carbon nanotubes (CNTs) were selected as the coating material. The responsivity of the detector is about $18.3 \mathrm{~V} / \mathrm{W}$ at $14 \mathrm{~Hz}$. The detectors were used to establish an electrically calibrated system with uncertainty less than $4 \%$ at room temperature. The relative deviation of measurement is less than $3 \%$ between $0.2 \mathrm{~W} / \mathrm{m}^{2}$ and $200 \mathrm{~W} / \mathrm{m}^{2}$, and the relative deviation is less than $5 \%$ between $0.002 \mathrm{~W} / \mathrm{m}^{2}$ and $0.02 \mathrm{~W} / \mathrm{m}^{2}$. Secondly, a $128 \times 1$ linear array was designed and fabricated. The responsivity of the linear array is $3.5 \times 10^{4} \mathrm{~V} / \mathrm{W}$ at $10 \mathrm{~Hz}$, and the noise equivalent radiant power (NEP) is $0.4 \mathrm{nW}$. The pyroelectric array and CMOS read-out circuit were electrically bonded by ultrasonic bonding process to be uncooled focal plane array (FPA). The FPA was tested under different radiation intensity. These results lay a foundation for the development of high-performance uncooled pyroelectric FPA based on novel ferroelectric single crystals.
\end{abstract}

Key words: PMN-PT, pyroelectric detector, finite element model, coatings, and uncooled FPA

\section{Introduction}

Pyroelectricity is an old phenomenon which was investigated at the turn of $20^{\text {th }}$ century. When there are modulated radiation which are absorbed by the front surface of pyroelectric detectors, there will originate temperature change which may cause a change in spontaneous polarization. This will produce a change of surface charges which can be sensed as a current by means of an external circuit between the front and back electrodes. The principle is shown in Fig. 1.
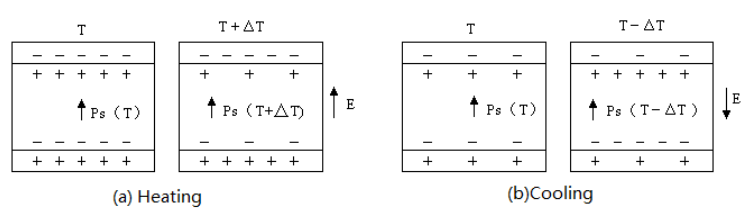

Fig. 1. Surface charges of pyroelectric detectors varied with temperature changing

Pyroelectric detectors have high sensitivity and work at at room temperature without cryogenic system. In addition, Pyroelectric detectors have comparatively flat spectral response in wide spectrum range and low cost. These merits make which are attractive to many civilian and military applications, such as driver night vision, security, fire detection, search and rescue, 
industrial, military, scientific, and medical applications. Recent investigations by Luo Haosu et al have exhibited that PMN-PT crystals are excellent pyroelectric materials with a high pyroelectric coefficient of $15.3 \times 10^{-4}$ $\mathrm{C} / \mathrm{m}^{2} \mathrm{~K}$ and a low thermal diffusivity of $3.7 \times 10^{-7}$ $\mathrm{m}^{2} / \mathrm{s}$ [1]. PMN-PT crystals show great potential as novel pyroelectric materials for infrared detectors and some related research has been carried out $[2,3]$.

In this paper, PMN-PT was selected as pyroelectric detector material. An electrically calibrated pyroelectric detector with special structure was developed. Electrically calibrated pyroelectric radiometer (ECPR) was first reported and originally developed by NIST scientists for optical power measurements over a broad wavelength range [4]. The originality of ECPR consists of the application of the electrical substitute method to the optical power measurements. The principle of ECPR is described in detail in Ref. [5]. It's an absolute measurement method and no standard sources are required for calibrations.

Then, a $128 \times 1$ linear array was designed and fabricated. The pyroelectric array and CMOS read-out circuit were electrically bonded by ultrasonic bonding process to be uncooled FPA. These results lay a foundation for the development of high-performance uncooled pyroelectric FPA based on novel PMN-PT single crystals.

\section{Electrically calibrated pyroelectric detectors}

The structure of electrically calibrated pyroelectric detectors is shown in Fig. 2. A finite element model was set up to optimize the structure of the detector and simulate the thermal diffusivity. According to the simulation results [6], the PMN-PT crystal is $18 \mathrm{~mm} \times 14$ $\mathrm{mm}$. The sensitive area is $10 \mathrm{~mm} \times 10 \mathrm{~mm}$ and the entrance aperture is $\Phi 8 \mathrm{~mm}$. The active area of radiation absorption is corresponding to the entrance aperture. The crystal was grinded and polished to $80 \mu \mathrm{m}$ in the thickness. Then, three $500 \mathrm{~nm}$ thick $\mathrm{Cr} / \mathrm{Au}$ electrodes and an insulating layer were deposited on the top surface and back surface of the crystal respectively. The insulating layer is polyimide with about $2 \mu \mathrm{m}$ of thickness.

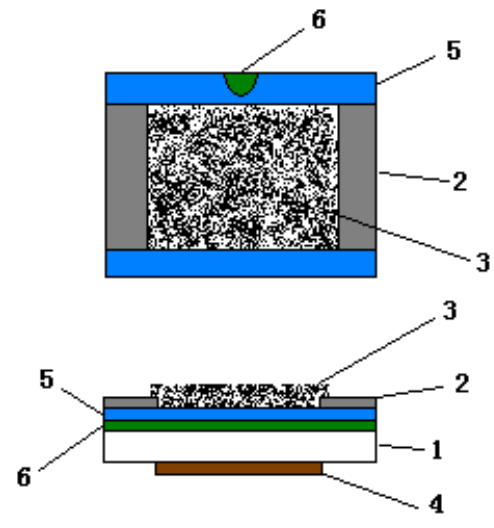

Fig. 2. Structure of electrically calibrated pyroelectric detectors (1)Pyroelectric material. (2)Cr/Au electrode for electrical power input. (3) Black coating. (4) $\mathrm{Cr} / \mathrm{Au}$ electrode for pyroelectric signal output. (5) Insulating layer. (6) Cr/Au electrode for ground connection.

There is a black coating on the surface of the pyroelectric detector which has good thermal contact with the pyroelectric transducer. At the same time, the coating is also electrically conductive. As shown in Fig. 3., both the radiation absorption and the electrically heating in the coating film can result in temperature change. Accordingly, the film can work both as optical absorber and electric heater.

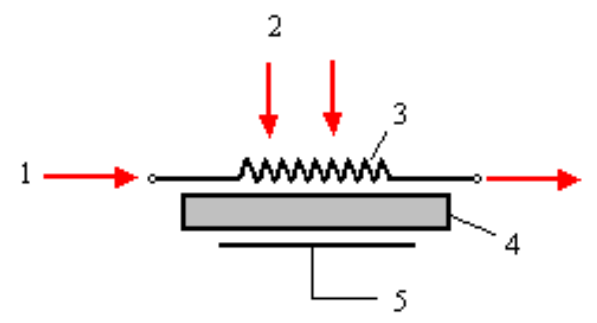

Fig. 3. The heating model of electrically calibrated detectors. (1)Electrical heating. (2)Radiant heating. (3)Black coating. (4)PMN-PT crystal. (5)Cr/Au electrode

Recent researches by John $\mathrm{H}$. Lehman et al have demonstrated that CNTs are good coatings on pyroelectric detectors as optical absorber $[7,8]$. A.C. Dillon et al have reported that CNTs have been to be promising candidate for the replacement of gold black coatings on pyroelectric detectors employed for laser power measurements [9]. In fact, CNTs also have good conductive properties which can work both as optical absorber and electric heater. In this paper, CNTs were selected as the coating material. The CNTs are commercially available multi-wall CNTs produced by chemical vapour deposition. The purity of the CNTs is higher than 95 wt.\% after treated by refluxing in high density nitric acid at 120 , and the specific surface area is about $233 \mathrm{~m}^{2} / \mathrm{g}$. The CNTs were dispersed to ethanol uniformly by 
sustained ultrasonic for 6 hours. Then, the solution was sprayed to the surface of the effective area (Fig. 4). The estimated thickness is about $1.5 \mu \mathrm{m}$. The sheet resistance of the coating is about $100 \Omega$. The infrared transmission spectrum was measured by Nicolet 6700 Spectrophotometers. As shown in Fig. 5, the transmissivity of the coating is less than $0.16 \%$ over a broad wavelength range from $1 \mu \mathrm{m}$ to $20 \mu \mathrm{m}$.

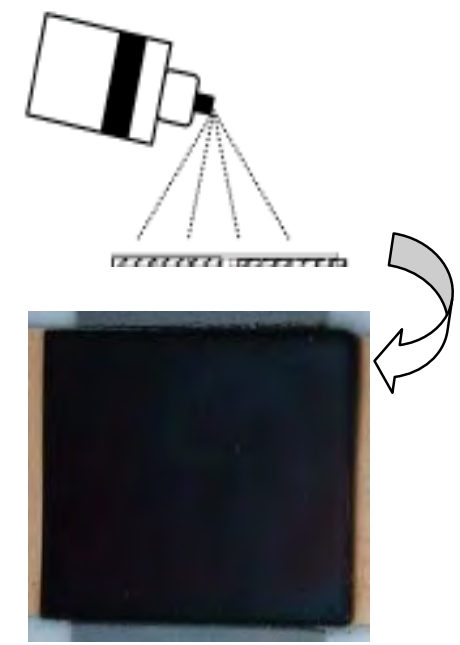

Fig. 4. Black coating was deposited on the sensitive area of the detector chip

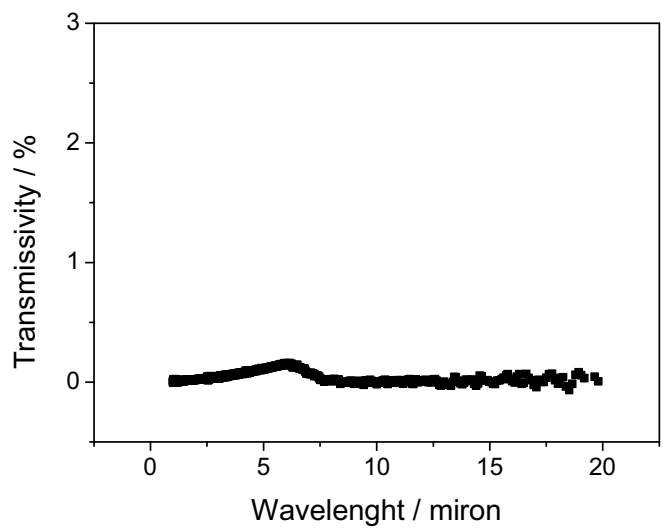

Fig. 5. The infrared transmission spectrum of CNTs coating

The detector was mounted in a metal package as shown in Fig. 6. The sensitive area of the pyroelectric chip is free-standing. The entrance is a circular aperture with a diameter of $8 \mathrm{~mm}$. The responsivity of the detector is about 18.3 V/W at $14 \mathrm{~Hz}$.

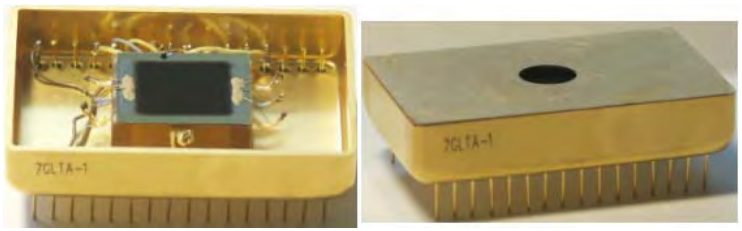

Fig. 6. Electrically calibrated pyroelectric detector coated with CNTs

The detectors were used to establish electrically calibrated radiometer by $\mathrm{Hu}$ Qi et $\mathrm{al}^{10}$. An electrical substitution method and autonulling circuit were adopted in the system. The errors of the electrical system are less than $0.5 \%$ [10], such as closed-up error and sampling error. According to analysis of the optical-electrical equivalence of the detector, the whole uncertainty of the system is less than $4 \%$. The radiometer was calibrated by Shanghai Institute of Measurement and Testing Technology (SIMT). The radiant source is a black-body at a temperature of $500 \mathrm{~K}$. The radiation was modulated to be square wave by an optical chopper with a frequency of $14 \mathrm{~Hz}$. The relative deviation of measurement is less than $3 \%$ between $0.2 \mathrm{~W} / \mathrm{m}^{2}$ and $200 \mathrm{~W} / \mathrm{m}^{2}$, and the relative deviation is less than $5 \%$ between $0.002 \mathrm{~W} / \mathrm{m}^{2}$ and $0.02 \mathrm{~W} / \mathrm{m}^{2}$.

\section{Focal plane array}

A $128 \times 1$ linear array was designed and fabricated based on PMN-PT. The $128 \times 1$ pyroelectric linear array adopts hybrid structure. Fig.7 shows the schematic view of the basic layout of $128 \times 1$ pyroelectric linear array. The sensitive area of each elements is $90 \mu \mathrm{m} \times 100$ $\mu \mathrm{m}$. The distance between adjacent elements is $100 \mu \mathrm{m}$. The first surface was grinded and polished. The top electrode was deposited on the chip after photolithography. Argon ion etching was applied for the isolation trench between adjacent elements. The width of the isolation trench is $10 \mu \mathrm{m}$. The detailed process of the fabrication is shown in Fig. 8, and Fig. 9 is a picture of the $128 \times 1$ pyroelectric chip. The isolation trench was displayed under microscope.

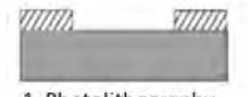

1. Photolithography after polishing the top surface

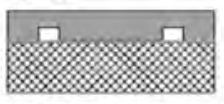

4. Grinding and polishing for the back surface

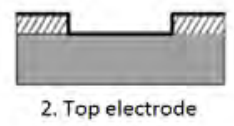

2. Top electrode

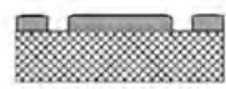

5. Back electrode Ion Beam $+1.1+1 .+\downarrow$

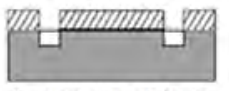

3. Argon ion etching for the isolation trench

PMN-PT VIII Photoresist

BS Sappire

- Flectrode

Fig. 8. Main fabrication process of pyroelectric linear array 


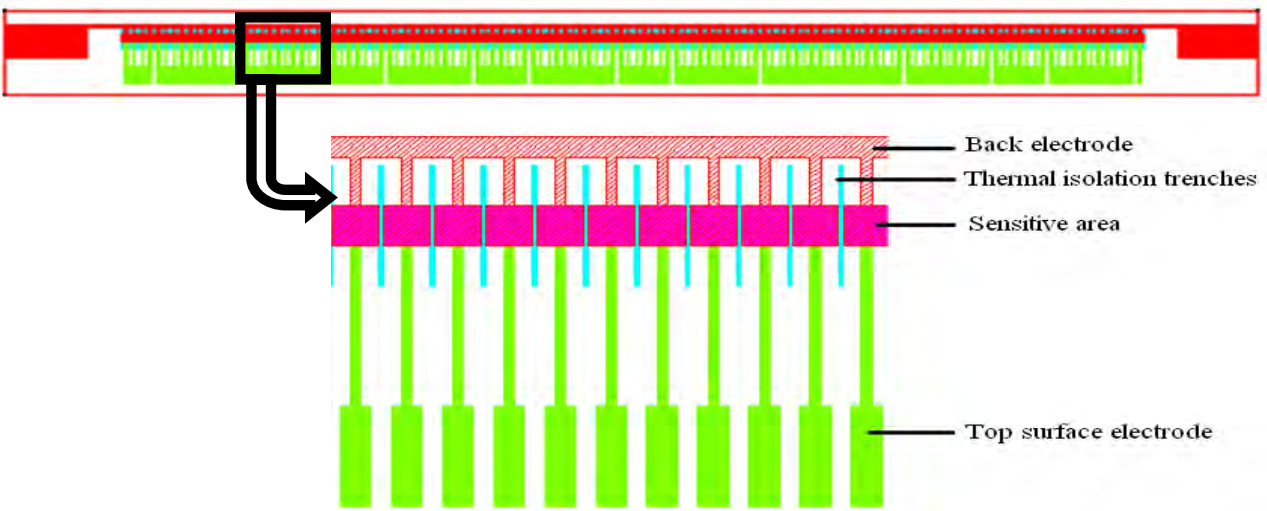

Fig. 7. The schematic view of the basic layout of $128 \times 1$ pyroelectric linear array

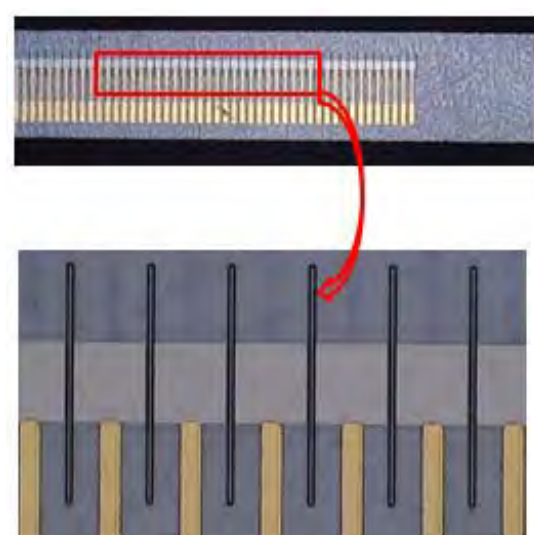

Fig. 9. A picture of pyroelectric chip

The pyroelectric chip is mounted on a support. But the sensitive area is free-standing in order to reduce the thermal conduction. The pyroelectric linear array was tested under different frequency. The detailed testing results are shown in Fig. 10 and Fig. 11. The responsivity of the linear array is $3.5 \times 10^{4} \mathrm{~V} / \mathrm{W}$ at $10 \mathrm{~Hz}$, and the NEP is $0.4 \mathrm{nW}$.

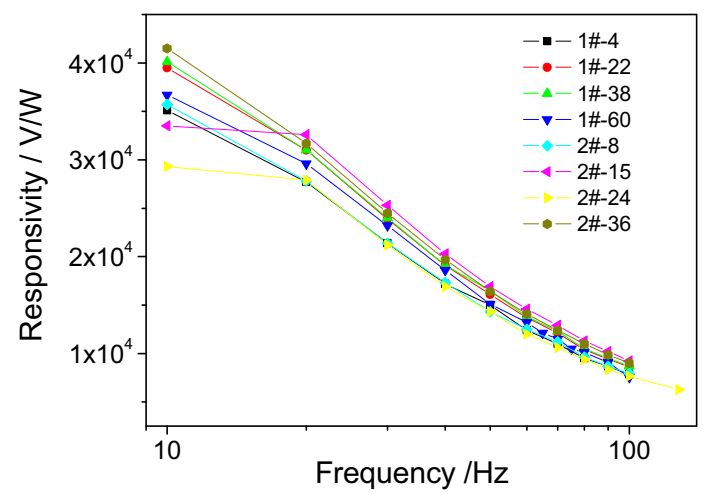

Fig. 10. The responsivity of the linear array under different frequency

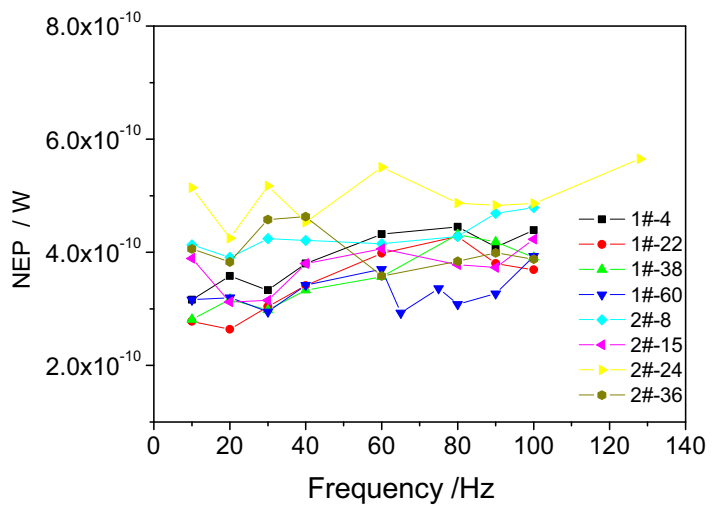

Fig. 11. The NEP of the linear array under different frequency

The pyroelectric array and CMOS read-out circuit were electrically bonded by ultrasonic bonding process to be uncooled FPA. Fig. 12 and Fig. 13 are the picture of the FPA.

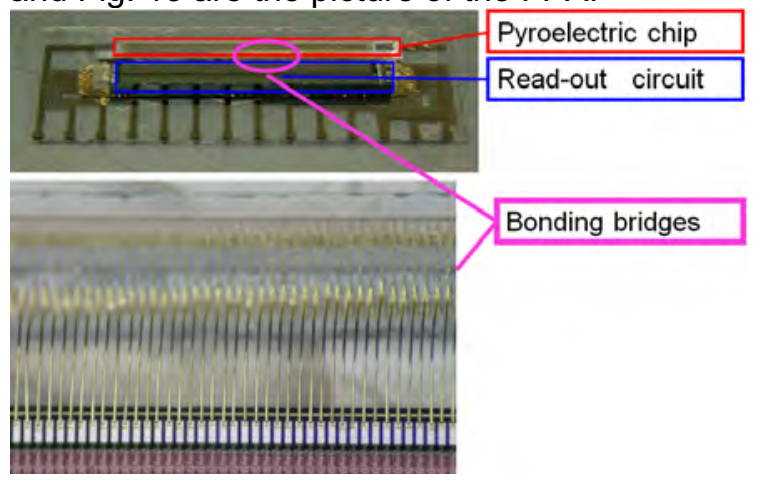

Fig. 12. A picture of FPA with bonding bridges

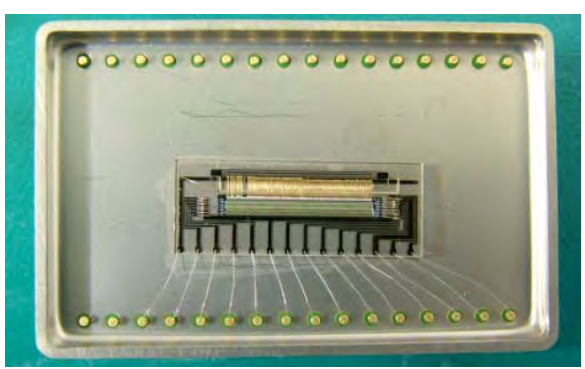

Fig. 13 A picture of FPA detector in metal package 


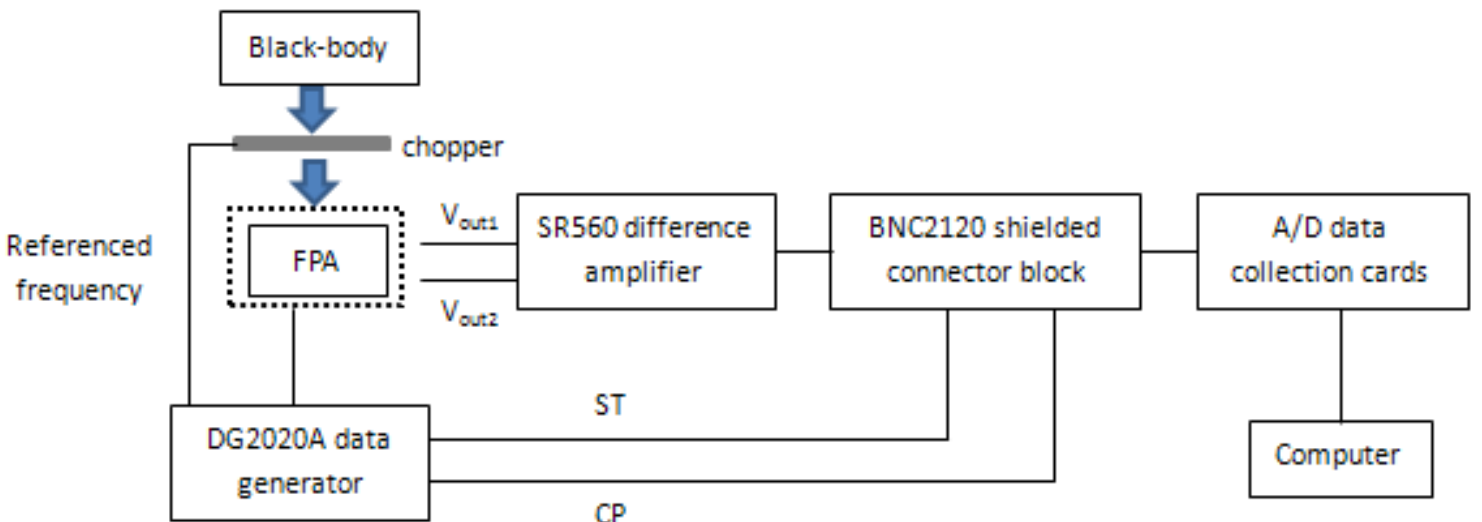

Fig. 14. The schematic of FPA testing system

The parameters of the FPA has been tested. The readout integration circuit (ROIC) is applied with capacitor feedback trans-impedance amplifier (CTIA) and correlated double sampling (CDS) structure which helps to reduce the noise of the circuit [11]. The integration capacitor (Cint) is $1 \mathrm{pF}$. Fig. 14 is the schematic of FPA testing system. As shown in Fig. 15, along with the increase of the power which was received by each element, the mean output of the FPA increase linearly. In this case, it states clearly that the output is the real pyroelectric signal of the FPA. The quantitative test of the FPA needs further research in the future.

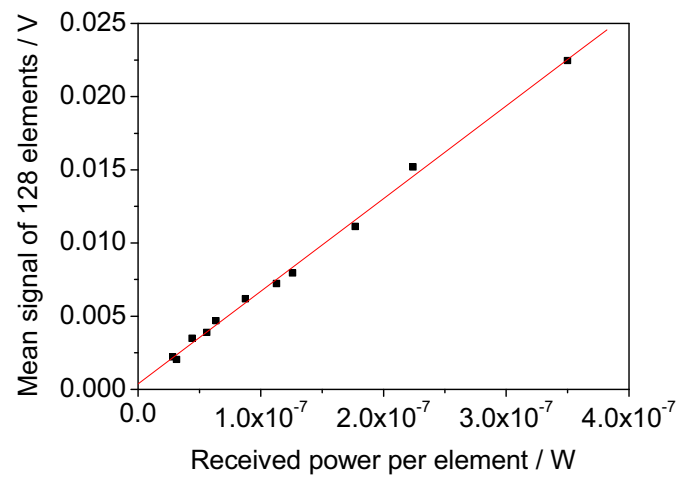

Fig. 15 The output of the pyroelectric FPA at room temperatue

\section{Conclusions}

PMN-PT was selected as pyroelectric detector material. An electrically calibrated pyroelectric detector with special structure was developed for calibration of radiation. CNTs were selected as the coating material. The responsivity of the detector is about $18.3 \mathrm{~V} / \mathrm{W}$ at $14 \mathrm{~Hz}$. The detectors were used to establish an electrically calibrated system with uncertainty less than $4 \%$ at room temperature. A $128 \times 1$ linear array was designed and fabricated. The responsivity of the linear array is $3.5 \times 10^{4} \mathrm{~V} / \mathrm{W}$ at $10 \mathrm{~Hz}$, and the NEP is $0.4 \mathrm{nW}$. The pyroelectric array and
CMOS read-out circuit were electrically bonded by ultrasonic bonding process to be uncooled FPA. The FPA was tested under different radiation intensity. These results lay a foundation for the development of highperformance uncooled pyroelectric FPA based on novel ferroelectric single crystals.

\section{Acknowledgements}

The authors wish to thank Prof. Luo Haosu (Shanghai Institute of Ceramics, CAS, China) for his supply of relaxor-based ferroelectric single crystals. We also acknowledge the national financial support: No. 60837003 and No. 60708028.

\section{References}

[1] Yanxue Tang, Xinming Wan, Xiangyong Zhao,et al., Large pyroelectric response in relaxor-based ferroelectric (1-x) $\mathrm{Pb}\left(\mathrm{Mg}_{1 / 3} \mathrm{Nb}_{2 / 3}\right) \mathrm{O}_{3}-\mathrm{xPbTiO}_{3}$ single crystals, Journal of Applied Physics 98, 084104 (2005); doi: 10.1063/1.2106014

[2] Yanxue Tang, Haosu Luo, High-performance pyroelectric single crystals for uncooled infrared detection applications, Infrared Physics \& Technology 52, 180-182 (2009); doi: 10.1016/j.infrared.2009.07.004

[3] Zhao, Xiangyong, Wu, Xiao, Liu, Linhua, et al., Pyroelectric performances of relaxor-based ferroelectric single crystals and related infrared detectors, Physica Status Solidi A: Applications and Materials 208, 1061-1067 (2011); doi: 10.1002/pssa.201000051

[4] Phelan, R.J. Jr., Cook, A.R., Electrically calibrated pyroelectric optical-radiation detector, Applied Optics 12, $2494-2500$ (1973); doi:

[5] Jon Geist, Harry J. Dewey, Michael A. Lind, Lowlevel periodic pulsed energy measurements with an electrically calibrated pyroelectric detector, Applied Physicds Letters 28, 171-173 (1976); doi:

[6] Shao Xiumei, Ding Jieying, Fang Jiaxiong, Design and thermal analysis of electrically calibrated pyroelectric detector, Infrared Physics 
\& Technology 55, 45-48 (2012); doi:

10.1016/j.infrared.2011.08.008

[7] John H. Lehman, Chaiwat Engtrakul, Thomas Gennett, Anne C. Dillon, Single-wall carbon nanotube coating on a pyroelectric detector, Applied Optics 44, 483-488(2005); doi: 10.1364/AO.44.000483

[8] Evangelos Theocharous, John Lehman, The evaluation of a pyroelectric detector with a sprayed carbon multi-wall nanotube black coating in the infrared, Infrared Physics \& Technology 54 34-38(2011); doi: 10.1016/j.infrared.2010.10.004

[9] Dillon, A.C., Mahan, A.H., Deshpande, R., et al., Hot-wire chemical vapor synthesis for a variety of nano-materials with novel applications, Thin Solid Films 501, 216-220(2006); doi:

10.1016/j.tsf.2005.07.182

[10] Hu Qi, Gu Lingjuan, Li Wen, Zhuang Songlin, Auto-nulling system design of ultraviolet enhanced electrically calibrated pyroelectric radiometer, Proceedings of SPIE 6150, 61504W(2006); doi: 10.1117/12.676750

[11] Li Xue, Huang Songlei, Chen Yu, et al, Noise characteristics of SWIR InGaAs linear FPAs, Journal of Applied Physics 112, 064509(2012); doi: 10.1063/1.4754579 